\title{
Collision Reduction Using Modified Q-Algorithm with Moving Readers in LED-ID System
}

\author{
$\mathrm{Vu}$ Van Huynh ${ }^{*}$ Associate Member, Nam-Tuan Le*, \\ Sunwoong Choi ${ }^{*}$, Yeong Min Jang ${ }^{*^{*}}$ Lifelong Members
}

\begin{abstract}
LED-ID (Light Emitting Diode - Identification) is one of the key technologies for identification, data transmission, and illumination simultaneously. This is the new paradigm in the identification technology environment. There are many issues are still now challenging to achieve high performance in LED-ID system. Collision issue is one of them. Actually this is the most significant issue in all identification system. LED-ID system also suffers from collision problem. In our system, collision occurs when two or more readers transmit data to tag at the same time or vice versa. There are many anti-collision protocols to resolve this problem; such as: Slotted ALOHA, Basic Frame Slotted ALOHA, Query Tree, Tree Splitting, and Q-Algorithm etc. In this paper, we propose modified Q-Algorithm to resolve collision at tag. The proposed protocol is based on Q-Algorithm and used the information of arrived readers to a tag from neighbor. The information includes transmitting slot number of readers and the number of readers that can be arrived in next slot. Our proposed protocol can reduce the numbers of collision slot and the successful time to identify all readers. In this paper our simulation and theoretical results are presented.
\end{abstract}

Key Words : Visible Light Communication (VLC), Light Emitting Diode Identification (LED-ID), Q-Algorithm.

\section{I . Introduction}

The traditional identification system is Barcode technique which is the oldest and stills exist to nowadays ${ }^{[1]}$. The data that uses in Barcode system is so small and can't be changed. The next generation of identification system is RFID (Radio Frequency Identification) technique which uses radio frequency to communicate and it is applied in many applications today. RFID can identify and get data quickly so it gradually replaces Barcode technique in the identification systems. The novel identification technique is LED-ID which is a new technique that uses communication via light waves (especially visible light) to contactless exchange data between tags and readers ${ }^{[2]}$. By using visible light communication (VLC) to communicate ${ }^{[3-5]}$, LED-ID has inherited many advances of this technique. LED-ID is very safe for human eyes and body comparing with radio frequency. The lighting systems already have existed in everywhere so we can apply LED-ID easily. The most advance of LED-ID is that this technique doesn't have interference with other systems or devices. Therefore, not only application that RFID have already exploited but also LED-ID can be applied in the special systems which RFID can't be used because of interference, such as: in hospital and space station. In every system, the most critical problem between tags and readers is collision.

In this paper, we only focus on the collision among readers. When two or more readers transmit

* This work was supported by the IT R\&D program of MKE/KEIT [10035362, Development of Home Network Technology based on LED-ID].

* Department of Electronics Engineering, Kookmin University, (yjang@kookmin.ac.kr), ( ${ }^{\circ}$ : corresponding author) 논문번호 : KICS2011-07-279, 접수일자 : 2011년 7월 4일, 최종논문접수일자 : 2012년 4월 24일 
their data simultaneously, this mounts of data will be collided at tag, which is typically called a collision problem. LED-ID system has one disadvantage which is the limitation of FOV (field of view) or the angle of LED. This limitation can increase number of collision because readers cannot sense the transmitting of others. So, we cannot apply CSMA-CA algorithm in LED-ID system to resolve this problem. The anti-collision protocol which is used in RFID system can solve the limitation of FOV in LED-ID system. In RFID system, there are many anti-collision protocols ${ }^{[6]}$. such as: Slotted ALOHA, Basic Frame Slotted ALOHA, Query Tree, Tree Splitting, Q-Algorithm etc. In these anti-collision protocols, and Q-Algorithm is used in EPC-global Generation-2 in the RFID standard, and there are some papers which are improved read rate [7] or considered capture effect in Q-Algorithm ${ }^{[8]}$. The selection method of effective $Q$ and $C$ parameters has been proposed $\mathrm{in}^{[9]}$. The modifying and optimizing of Q-algorithm parameters also presented in $\left[{ }^{10]}\right.$. Q-Algorithm updates the frame size through $Q$ value slot-by-slot according to the status of the preceding received slot. This algorithm is very effective and can be applied in every system, that why we use Q-Algorithm to solve the collision problem in LED-ID system. The above papers have not considered about the movement of readers yet. We consider the movement of readers in this paper and apply in LED-ID system. In LED-ID system, we propose modified Q-Algorithm which all readers are identified quickly and reduce the probability of collision slot. This algorithm has an outstanding performance and efficient to apply in the LED-ID system.

The rest of this paper is organized as follows. First, section II overviews LED-ID system and system architecture; second, section III proposes a new anti-collision protocol called modified Q-Algorithm; thirst, section IV describes numerical results and finally section $\mathrm{V}$ presents conclusion of this paper.

\section{Overview LED-ID System and System}

\section{Architecture}

\subsection{LED-ID system}

In this part, we will introduce the most fundamental LED-ID system, which has already mentioned in ${ }^{[2]}$. LED-ID system is used visible light as a transmission medium between readers and tags to communicate. Reader is the user that needs information and tag is a LED-ID that supports information for reader. As Fig. 1, tags in LED-ID system are fixed system and they connect with database server to support streaming data for readers. Readers are considered as users which are moving in the LED-ID system and need information from tags. The processing of LED-ID system includes two phase. Firstly, identification phase is the period which tag is identified ID of readers and the readers send request information for tag. Based on request information of readers, tag will allocate them into one or more slot in the data transmission phase. Secondly, after identification phase, readers will move to data transmission phase which tag will support data stream as the request of reader. In second phase, each reader will receive one or more slots for transmitting data. The number of slots that each reader occupies in data transmission period is depended on its kind of data transmission and the QoS (quality of service).

Now, we will introduce more about the process between tag and reader in LED-ID system. When reader comes into the range of tag, reader will listen to the query command from the tag. Then, reader sends ID and request information to tag in a random slot. If there is more than one reader sending in that slot, collision occurs at tag. When collision occurs, tag will send another query command and reader will resend ID in the next round. If only one reader sends ID and request information in one slot, reader will be identified successfully. Then, reader move to data transmission phase to transmitting data. This is just overview the process of LED-ID system. In the next part we will introduce detail the architecture of proposed algorithm.

\subsection{System architecture}


In this part, we'd like to discuss about system architecture of proposed algorithm. Fig. 1 shows the assumption scenario architecture. We can see this scenario in some applications such as: museum, exhibition etc. In these types of application scenario, tags are arranged sequentially as described in Fig. 1. Readers only have one way to go into the system and one way to go out the system. Therefore, normally readers pass through tags sequentially. But there are still some cases, readers skip next tag and move to another tag. We assume that tags can make neighbor relationship with near tags. For example, tag\#2 is neighbor with tag\#1 and tag\#3. Tag also can exchange information with neighbor tags. When readers move out the range of tag $i^{\text {th }}$ they will come to the range of tag $(i+1)^{t h}$. In this case, readers have already identified at tag $i^{\text {th }}$ and they join arrived reader group at tag $(i+1)^{\text {th }}$ as showing in Fig. 1 and Fig. 2; therefore, tag $i^{\text {th }}$ will inform for tag $(i+1)^{\text {th }}$ the information of arrived reader. The information that tag $i^{\text {th }}$ supply for tag $(i+1)^{\text {th }}$ includes reader ID and slot number which reader uses in tag $i^{\text {th }}$. Based on that information, tag $(i+1)^{\text {th }}$ will allocate arrived reader in the given slot in identification phase. So, arrived reader can be identified successfully and send request data with collision free and quickly. After that, arrived reader will move to transmission phase to receive data streaming from tag. But when readers do not pass through tags sequentially, readers will joint in the unidentified reader group and target tag has to identify them again. It is mean that readers move out the range of tag $i^{t h}$ and come into the range of tag $(i+1+j)^{\text {th }}$ instead of tag $(i+1)^{t h}$. But tag $(i+1)^{\text {th }}$ has already received information of moving reader from tag $i^{\text {th }}$, this is a limitation of proposed algorithm.

The classification of reader in the range of one tag is present in Fig. 2 . Fig. 2 is classified reader into two groups which are unidentified reader and identified reader. Unidentified reader is the group of readers in the range of tag which have not been identified yet. Identified reader is the group of readers in the range of tag which tag has already known the ID. In the identified reader type, we also divide into two groups, which are arrived reader and already identified reader. Already identified reader is the group of readers which the tag has already known ID by previous identified process. Arrived reader is the group of readers which the tag has known the ID by received information from neighbor tag.

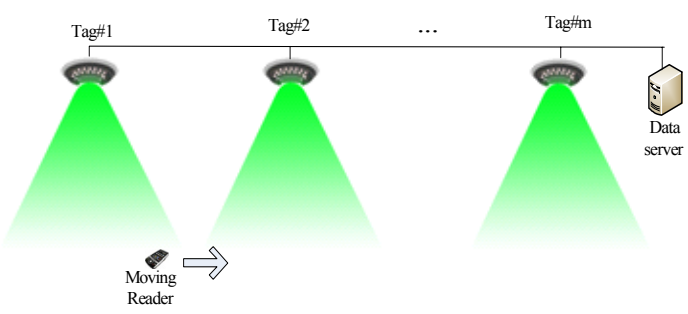

Fig. 1. Assumption scenario architecture.

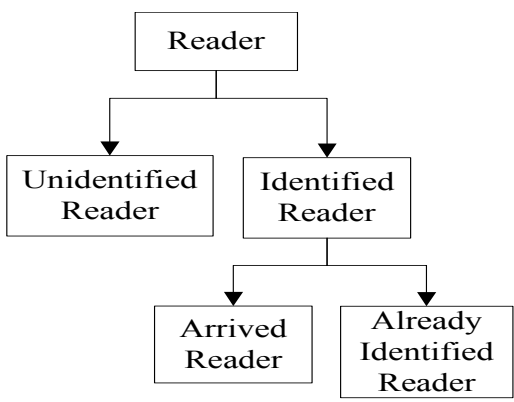

Fig. 2. Classification of readers in the range of one tag.

\section{Proposed Modified Q-Algorithm for LED-ID Anti-collision Protocol}

\subsection{Q-Algorithm in EPC-global Genaration-2}

First, we discuss about the operation of Q-Algorithm and then we will go to the approach of modified Q-Algorithm. Q-Algorithm is an anti-collision protocol which is used in EPC-global Generation-2 in the RFID standard. The process of this algorithm is described in the Fig. 3. In the Fig. 3 we can see that the tag uses 3 types of query command (Query, QueryRep, QueryAdjust) to identify all readers in its range. To start the identification process, the tag issues the Query command which includes the $Q$ value. When readers receive the Query command, readers will generate a random number in the range $\left(0,2^{Q}-1\right)$. These values will become the slot number that readers use to transmit there ID to the tag. If the readers generate the value 0 , they can transmit $R N 16$ in the 
next slot. Otherwise, they have to wait until their slot number by 0 . The QueryAdjust is issued when the $Q$ value is changed by the tag and the QueryAdjust only is used after the collision or idle slot. All readers will generate a new slot number when they receive the new $Q$ value (including in QueryAdjust command) from the tag. In the three types of query, only QueryRep does not have the $Q$ value. The QueryRep only has one purpose which is decreased the slot number of remaining readers by 1. Q-Algorithm is not considered about the movement of readers. When some readers which are identified by one tag move to another tag, these readers will be identified again in the range of new tag. To avoid this case, we will introduce modified Q-Algorithm in the next part.

In this subsection, we want to introduce the algorithm to adjust the $Q$ value. This algorithm is presented in Fig. 4 and it is applied in EPCglobal Gen-2. Fig. 4 shows the parameter $Q$ which is an integer value in the range $(0: 15)$ and it is round by $Q_{f p}$ value, with $Q_{f p}$ is a floating-point representation of $Q$. The new $Q$ value is determined by the $C$ value (with $0.1<C<0.5$ ) and the $Q_{f p}$. The $Q_{f p}$ only changes when we have a collision slot or idle slot. The $Q_{f p}$ will add with $C$ if collision occurs or $Q_{f p}$ will minus with $C$ if we have idle slot. The tag uses small values of $C$ when parameter $Q$ is large or large values of $C$ when parameter $Q$ is small. So, after one slot the parameter $Q$ can increase by 1 , decrease by 1 or no change.

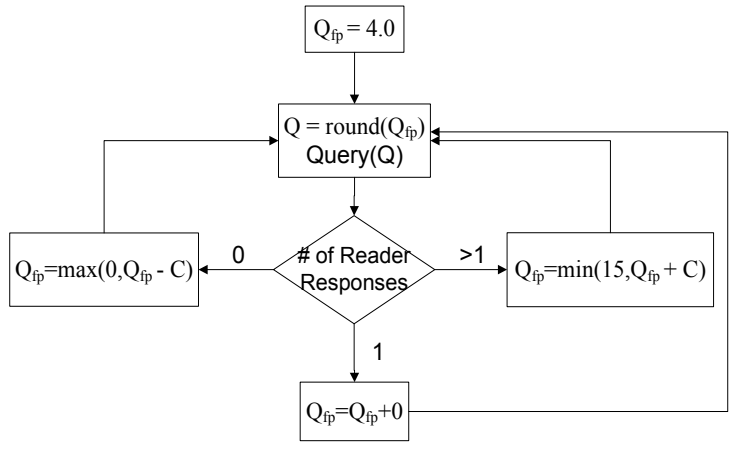

Fig. 4. Traditional algorithm for choosing the parameter Q.

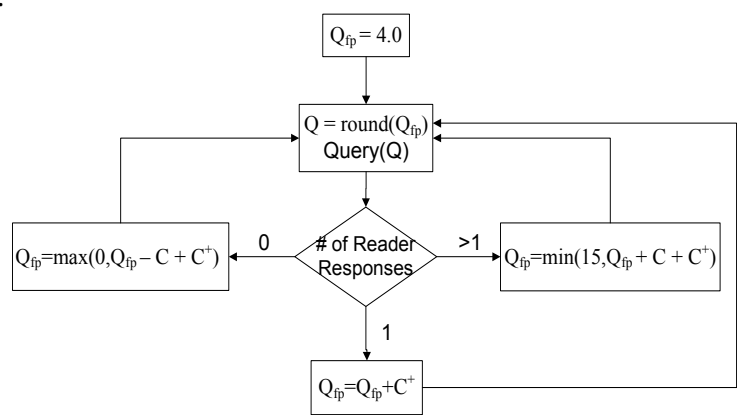

Fig. 5. Modified Q-Algorithm for choosing the parameter Q.

\subsection{Description of modified Q-Algorithm}

In the previous part, we have already mentioned about Q-Algorithm; now, we introduce proposed modified Q-Algorithm. In proposed algorithm, we consider readers move from tag $i^{\text {th }}$ to its neighbor $\operatorname{tag}(i+1)^{\text {th }}$.

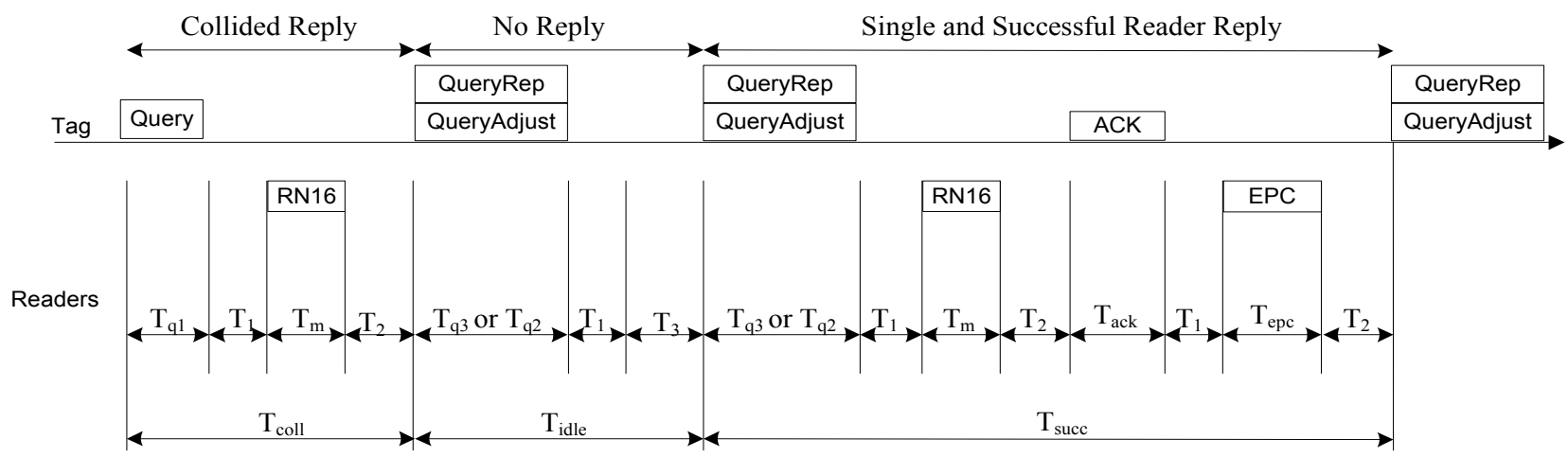

Fig. 3. The identification process. 
We assume that tags can make neighbor relationship with near tags. Tag also can exchange information with neighbor tags. The information that $\operatorname{tag} i^{\text {th }}$ supplies for tag $(i+1)^{\text {th }}$ includes reader ID and slot number which reader uses in tag $i^{\text {th }}$. Based on that information, tag $(i+1)^{\text {th }}$ will allocate arrived reader in the given slot in identification phase.

In addition, tag $(i+1)^{\text {th }}$ bases on the number of arrived readers which can be come in the next slot to determine the frame size.

In Fig. 5, we have a new value to calculate parameter $Q$ which is $C^{+}$as defining below:

$$
C^{+}=\log _{2}\left(2^{Q_{f p}}+A_{r}\right)-Q_{f p}
$$

$A_{r}$ is the number of arrived readers which can be come in the range of tag in the next slot. The ID of $A_{r}$ readers have already identified by neighbor tags, and it will be informed for tag when these readers come. $Q_{f p}$ is the floating-point to determine the $Q$ value.

The following part demonstrates the equation (1):

When tag receives $A_{r}$ arrived readers which the tag has already known the information. The number of readers in the range of tag is calculated:

$$
n_{\text {new }}=n_{\text {old }}+A_{r}=2^{Q_{f p}}+A_{r}
$$

where $n_{\text {new }}=2^{Q-n e w} Q_{-}$new $=Q_{f p}+C^{+}$. Therefore we have equation (3) from equation (2):

$$
2^{Q_{f p}+C^{+}}=2^{Q_{f p}}+A_{r}
$$

After applying logarithm in equation (3), the equation (1) is proved.

The operation of algorithm in Fig. 5 is similar with Q-Algorithm, it only different at the value of parameter $\mathrm{Q}$ when we have the movement readers in the system.

\subsection{Analysis of modified Q-Algorithm}

In this subsection we analysis the performance of new algorithm. We compare two aspects of modified
Q-Algorithm with original Q-Algorithm which are the probability of collision in one slot and the time to identify one reader successfully.

The frame size is not fixed and it can be changed after each slot to adapt with the current mount of readers in the range of tag. We assume that the frame size is $N$ slots $\left(N=2^{Q}\right)$, the numbers of readers are $n . k$ is the number of readers simultaneous transmitting in one slot $(0 \leq k \leq n)$. We have the probability of $k$ :

$$
P(k)=\left(\begin{array}{l}
n \\
k
\end{array}\right)\left(\frac{1}{N}\right)^{k}\left(1-\frac{1}{N}\right)^{n-k}
$$

From (1), when $k=0$ we have the probability of idle slot:

$$
P_{\text {idle }}=\left(\begin{array}{l}
n \\
0
\end{array}\right)\left(\frac{1}{N}\right)^{0}\left(1-\frac{1}{N}\right)^{n-0}=\left(1-\frac{1}{N}\right)^{n}
$$

From (1), when $k=1$ we have the probability of successful slot, or readable slot:

$$
P_{\text {succ }}=\left(\begin{array}{l}
n \\
1
\end{array}\right)\left(\frac{1}{N}\right)^{1}\left(1-\frac{1}{N}\right)^{n-1}=\frac{n}{N}\left(1-\frac{1}{N}\right)^{n-1}
$$

Based on (1), (2) and (3), we can calculate the probability of collision slot:

$$
\begin{aligned}
P_{\text {coll }}=1-P_{\text {idle }}-P_{\text {succ }} & =1-\left(1-\frac{1}{N}\right)^{n}-\frac{n}{N}\left(1-\frac{1}{N}\right)^{n-1} \\
& =1-\left(1-\frac{1}{N}\right)^{n-1}\left(1-\frac{n+1}{N}\right)
\end{aligned}
$$

Equation (4) is the general equation to calculate the probability of collision slot. Now, we will calculate the probability of collision slot in the case of proposed algorithm. We have the new frame size is $N=2^{Q \text { new }}$, and $A_{r}$ is the number of arrived readers which can be arrived in the next slot. Tag will allocate $A_{r}$ arrived readers in $A_{r}$ given slots to communicate; therefore, the remaining slots $2^{Q_{-} \text {new }}$ $A_{r}$ will reserve the slots for unidentified readers $\left(n_{u}\right)$ to identify. So, we have the probability of collision 
slot between unidentified readers in the remaining slots:

$P_{\text {coll_u }}=1-\left(1-\frac{1}{2^{Q_{-} \text {new }}-A_{r}}\right)^{n_{u}-1}\left(1-\frac{n_{u}+1}{2^{Q_{-} \text {new }}-A_{r}}\right)$

Based on (8) we can calculate the probability of collision slot in frame:

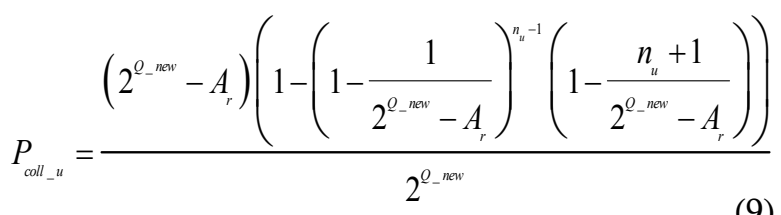

\section{Numerical Results}

In this part, we validate the proposed algorithm and compare the result with original algorithm. We have simulated our work using Matlab version 7.0 and attempts are made in the simulation to emulate the LED-ID system. The simulations base on the parameters which are listed in Table 1.

Table 1. Simulation parameters

\begin{tabular}{|l|l|}
\hline Parameter & Value \\
\hline Initial $Q_{f t}$ & 4 \\
\hline Maximum Q value & 15 \\
\hline Maximum number of readers & 500 \\
\hline $\begin{array}{l}\text { Distance between Tag I and } \\
\text { Tag 2 }\end{array}$ & $4 m$ \\
\hline $\begin{array}{l}\text { Vertical Distance between } \\
\text { Reader and Tag }\end{array}$ & $1 \mathrm{~m}$ \\
\hline PHY data rate & $24.4 \mathrm{~kb} / \mathrm{s}$ \\
\hline Concentrator FOV & $60($ degree $)$ \\
\hline Optical clock rate & $200 \mathrm{kHz}$ \\
\hline
\end{tabular}

In the simulation, maximum numbers of readers are 500 in the range of tag. In addition, we set some parameters, the initial $Q_{f t}$ value is 4 and $r$ is a value based on this ratio with $r=A_{r} / n$. The value of $r$ is very important, if $r$ is larger the modified Q-Algorithm presents more effective. In order to compare Q-Algorithm and modified Q-Algorithm, we consider the case with $r=0.1$ in Fig. 6 and Fig. 7. With a small value of $r$, we also can see the advantage of our proposed scheme. According to the variation of $r$ value, we can see the effective of modified Q-algorithm in Fig. 8 and Fig. 9 when $r$ value is increased. We consider two important parameters in every anti-collision protocol in our numerical results which are probability of collision and the total number of slots consuming.

Probability of collision: this parameter is the probability which one slot can be collided. Probability of collision is a critical metric to effect the efficient of system. Fig. 6 and Fig. 8 show the numerical results about the probability of collision. In Fig. 6, the comparison between Q-Algorithm and modified Q-Algorithm about probability of collision is presented by analytical results and simulation results. We can observe that the modified Q-Algorithm has a better performance than Q-Algorithm in Fig. 6 with $r=0.1$. We use $r=0.1$ in this plot to show that with a small value of $r$, the modified Q-Algorithm can achieve better performance than Q-Algorithm. When the numbers of reader are too small, the performance is not clear with $r=0.1$; but when the numbers of reader are large we can easy recognize the efficiency of proposed algorithm. In Fig. 6, we also see the well match of analytical results and simulation results. In Fig. 8, we show the performance of modified Q-algorithm with variation of $r$ value. The parameter $r$ increases, the probability of collision decreases also. The best efficient of proposed algorithm can be achieve in the case that total number of readers are arrived readers.

The number of slots: this metric is the total slots which we use to identify all readers. This metric also is the delay time to recognize all readers. The fast identification is the most significant factor in anti-collision protocol. Fig. 7 and 9 show the total number of slots which is used to identify successfully all readers. In Fig. 7, we can see that the proposed algorithm can identify all readers faster than original algorithm with $r=0.1$. With different value of $r$, the simulation results of proposed algorithm are presented in Fig. 9. When $r$ value is increased the total number of slots is decreased as well. The best performance of modified Q-algorithm is achieved when $r=1$ or when all readers are the 
arrived readers.

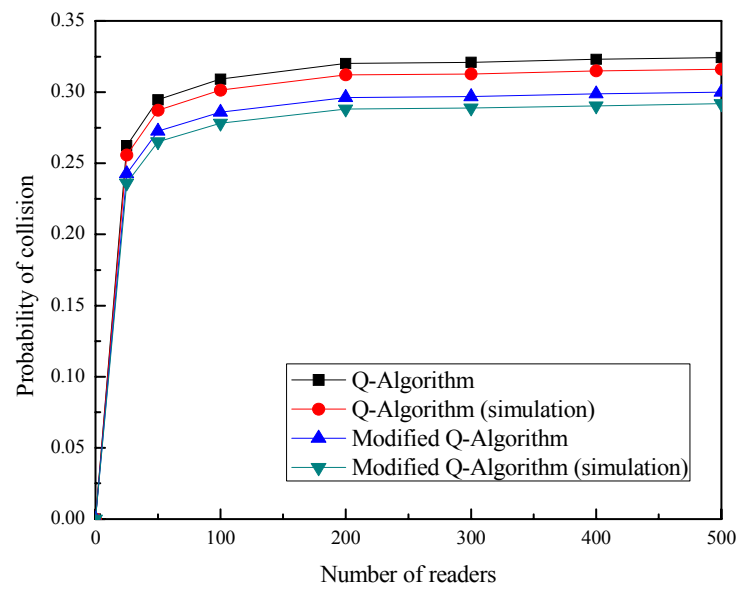

Fig. 6. Probability of collision in one slot $(r=0.1$ case).

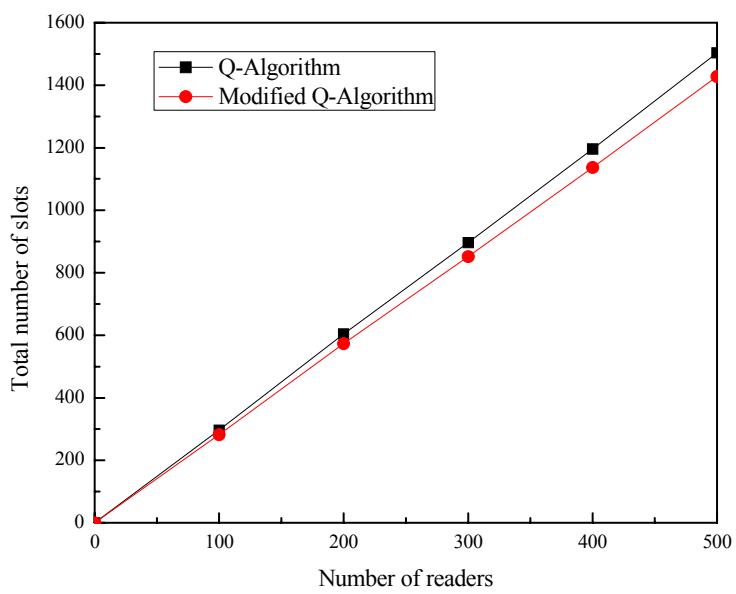

Fig. 7. Total number of slots to identify all readers (Simulation result in $\mathrm{r}=0.1$ case).

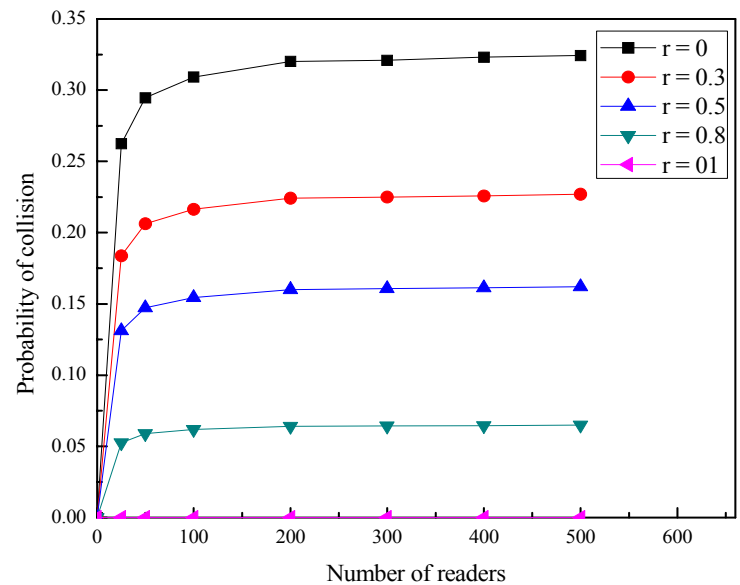

Fig. 8. Probability of collision in one slot for modified Q-Algorithm.

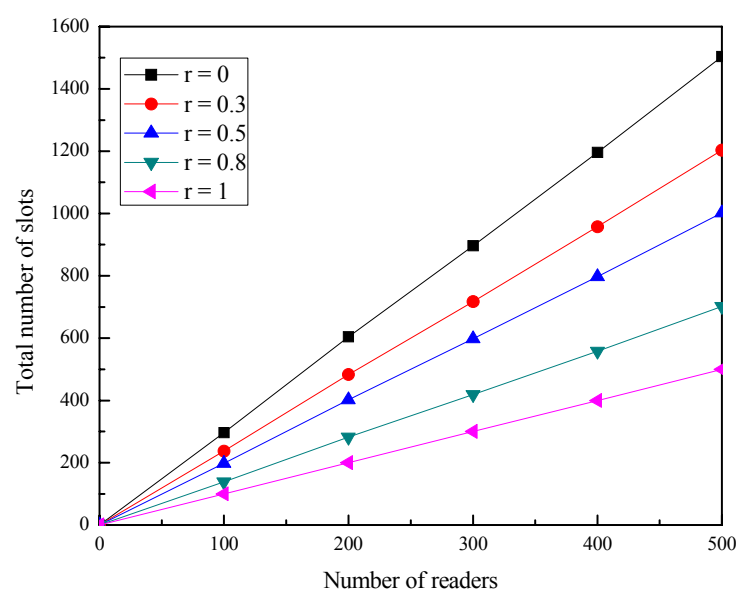

Fig. 9. Total number of slots for modified Q-Algorithm.

\section{Conclusion}

LED-ID will become popular in the near future, and anti-collision protocol for LED-ID is a one of critical issues. Modified Q-Algorithm is an enhancement of Q-Algorithm to apply in LED-ID system. This algorithm has considered about the movement of reader between tags and exploited the information which obtains from neighbor tag to increase the performance. The numerical results verified the outstanding of modified Q-Algorithm compare with Q-Algorithm. Not only increasing the efficient of Q-Algorithm, this paper also proposes the new process for successful slot in Q-Algorithm. Through numerical results, we have already proved the efficient of proposed algorithm by two parameters which are total number of slots and the probability of collision.

\section{References}

〔1〕 H. E. Burkc, A. V. N. R. iihol, Handbook of Barcoding systcms, 1984.

〔2〕 I. H. Park, Y. H. Kim, J. Cha, K. Lee, Y. M. Jang and J. Y. Kim, "Scalable optical relay for LED-ID systems," International Conference on Technology Convergence (ICTC), 2010.

(3] J. Y. Kim, "LED Visible Light Communication Systems," Hongreung Science Publishers, Seoul, Korea, 2009.

[4] T. Mukai and S. Nakamura, "White and W 
LEDs," OYO BUTURI, vol.68, no. 2, pp.152-155, 1999. G.

[5] T. Taguchi, "Technological innovation of high-brightness light emitting diodes (LEDs) and a view of white LED lighting system," OPTRONICS, vol. 19, no.228, pp.113-119, 2000.

[6] D. K. Klair, K. W. Chin and R. Raad, "A survey and tutorial of RFID anti-collision protocols," Communications survey \& tutorials, IEEE, pp. 400, 2010.

[7] Y. Maguire and R. Pappu, "An optimal Q-Algorithm for the ISO 1800-6C RFID protocol," Automation Science and Engineering, IEEE Transactionson, 2009.

[8] Li, Bo, Wang, and Junyu, "Efficient anti-collision algorithm utilizing the capture effect for ISO 1800-6C RFID protocol," Communications Letters, IEEE, pp 352, 2011.

[9] Y. J. Lee, D. K. Kwon, and H. N. Kim, "Improvement of collision-arbitration for ISO 1800-6 type C RFID system", ITC-CSCC, Vol. 2, 2006.

[10] D. Lee, K. Kim, and W. Lee, " $\mathrm{Q}^{+}$-Algorithm: an enhanced RFID tag collision arbitration algorithm", Ubiquitous intelligence and computing, Vol. 4611, 2007.

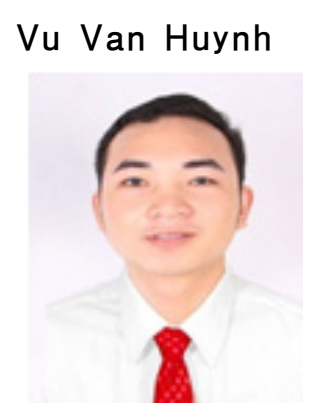

He received the diploma in Faculty of information and communication Technology from HaNoi University of Science and Technology, Viet Nam in 2010. He is currently Master student in Department. Electronics Engineering, Kookmin University, Korea. His research interests are visiblelight communication, MAC protocol, RFID, LED-ID, and localization based service.

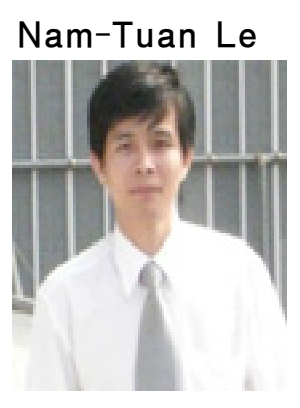

He received the diploma in Faculty of information and communication Technology from HaNoi University of Science and Technology, Viet Nam in 2005. $\mathrm{He}$ received the M.Sc. in Electronics Engineering from Kookmin University, Korea in 2010. He is currently Ph.D. student in Department Electronics Engineering, Kookmin University, Korea. His research interests are sensor networks, MAC, queuing model, resource management, and visible light communication.

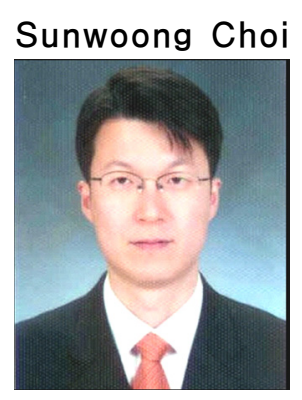

He received the B.S., M.S., and Ph.D. degree in Computer Engineering from Seoul National University, Korea, in1998 and 2000, and 2005, respectively. He worked for Samsung

Electronics between 2005 and 2007. Since 2007, he is with the Schoolof Electrical Engineering, Kookmin University, South Korea. His research interests include VLC MAC and routing protocols for mobile wireless networks, network resource management, and future internet. 


\section{Yeong Min Jang}

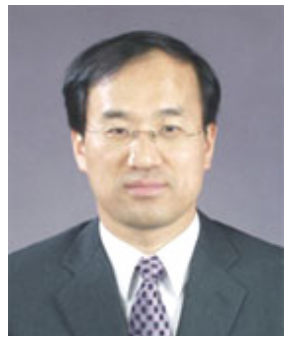

$\mathrm{He}$ received the B.E. and M.E. degree in Electronics Engineering from Kyungpook National University, Korea, in 1985 and 1987, respectively. $\mathrm{He}$ received the doctoral degree in Computer Science from the University of Massachusetts, USA, in 1999. He worked for ETRI between 1987 and 2000. Since Sept. 2002, he is with the School of Electrical Engineering, Kookmin University, Korea. His research interests are 5G Mobile, radio resource management, LED-ID, VLC networks, multi-screen service, and convergence networks. 